\title{
TOWARDS A METHODOLOGY TO MEASURE THE ALIGNMENT OF VALUE SYSTEMS IN COLLABORATIVE NETWORKS
}

\author{
António Abreu, Patrícia Macedo, L.M. Camarinha-Matos \\ New University of Lisbon \\ Quinta da Torre - 2829-516 Monte Caparica, PORTUGAL \\ ajfa@fct.unl.pt,.pmacedo@est.ips.pt,.cam@uninova.pt,
}

\begin{abstract}
As networks are typically formed by heterogeneous and autonomous entities, it is natural that each member has its own set of values. As a result, the ability to quickly identify partners with compatible or common values represents an important element for the success of collaborative networks. However, tools to measure the level of alignment are lacking. Applying some soft modeling tools, this paper discusses some perspectives and criteria to measure the level of alignment among a set of members in the context of a VO breeding environment.
\end{abstract}

\section{INTRODUCTION}

A number of requirements are needed to create successful collaborative coalitions, including: sharing of goals among members, having some level of mutual trust, having created some common infrastructures, and having agreed, totally or partially, on some practices and values.

Conciliating different organizational values is a challenge faced by collaborating organizations in our days. In general, the structure of a value system, and therefore the drivers of the Collaborative Networked Organizations' (CNO) behavior, includes multiple variables / aspects. Complementarily there are other elements that strongly influence or determine the behavior of a $\mathrm{CNO}$ and its members, such as the schema of incentives, trust building and management mechanisms, ethical code, the CNO culture, and the contracts and collaboration agreements.

Since collaborative networks are typically formed by heterogeneous and autonomous entities, it is natural that each member has its own set of values, and thus conflicts among partners might emerge due to existence of values misalignment (Abreu,2006). On the other hand, it is often assumed that the alignment between values systems of members involved in collaborative processes is a pre-requisite for a successfully co-working. However, the concept of alignment of value systems is difficult to define. Nevertheless, it can be intuitively understood that when the values of one member are incompatible with the values of another, there is a misalignment and the potential for conflicts is high. When the values of a member are compatible with the values of another member, there is an alignment and the potential for emergence of conflicts is lower.

The existence of a total alignment does not imply the total elimination of conflicts. An assessment of the level of alignment thus rather implies that the causes for conflicts are better understood and thus mechanisms may be designed for the progressive resolution of 
problems. Consequently, the level of alignment might work as a predictive indicator of the potential level for collaboration and also the capacity that a coalition has for getting agreements when conflicts take place during a collaborative process. The ability to quickly identify partners with a strong alignment represents an important boosting element for successful coalition formation.

Furthermore, the measurement of the level of alignment depends on the criteria that are used. In fact, there are several aspects that must be considered when we try to measure the level of alignment between two value systems. In some contexts, the alignment can be related to the existence of common values, level of shared interests or aims (ethical principles, business practices, etc...). On the other hand, there are some cases in which the level of alignment must be related to the existence of complementary values, relations among values, etc.

As the measurement of the level of alignment depends on several aspects that have an imprecise description, the adoption of soft modeling perspectives and techniques seem promising. Therefore, this paper suggests some perspectives to measure and analyze the level of alignment between value systems in a CNO context based on soft modeling techniques.

\section{SOME BACKGROUND}

In psychology and sociology values have typically been conceptualized as shared beliefs about desired behaviors and end-states, as in (Rokeach,1973). These shared beliefs concern the processes of goal pursuit and outcomes. Merton advocates that cultural objectives of an organizational unit are the "Things worth striving for" - what has outcome value in the culture (Merton,1957). Value has also been defined as the "relative worth, utility, or importance: degree of excellence". This definition of value highlights the fact that object's value depends on the "standard" that is used in the evaluation. Depending on the standard, the same object will be evaluated differently. The set of values hold by an individual or society define its value system.

This concept of value system has been studied and applied by diverse researchers. The philosopher Robert Hartman developed a formal Axiology, that is a branch of axiology (axiology is a general theory/science of human values, their origins, interrelations and dynamics) that attempt to use mathematical formalism to define values and value systems. Hartman (Hartman,1973) first defined the concept of value in terms of a logic-based axiom stating that value can be objectively determined according to a one-to-one correspondence between the properties of a given object and the meaning specifications contained in its concept. An object has value to the degree it fulfills its concept (Mefford,1997). Hartman introduced also the concept of dimension of value and developed the basic axioms for this concept. He defines three dimensions for value: Systematic Value, Extrinsic Value and Intrinsic Value. Hartman defends that the foundation concepts of axiology provide the framework for understanding an object's value and its valuations in precise terms of the three dimensions and their relationships to each other.

Goguen et Linde have developed, since 1978, several studies about value and value system in organizations (Goguen,2003) They proposed a method for using discourse analysis to determine a value system for an organization from a collection of stories told by members of the organization among themselves on informal occasions. The evaluative material collected from the stories is classified and represented using a formal structure called a value system tree. A value system tree serves as a formal summary of the interpretation that the analysts made from the collected data. 
Another contribution to the study of values systems came from the Distributed Artificial Intelligence discipline, where some theories where developed using agents. (Filipe, 2003) proposed an approach based on organizational agents where it is assumed that an agent is responsible for its values. The agent's preferences with respect to norms are defined in its value system, using deontic logic. In this approach an agent can represent a member of an organization or an organization itself. Another work (Woods,2003) proposed the use of par consistent logic to reason over values.

Gordijn, Yao-Huan Tan and Kartseva (Gordijn,2000), (Tan,2004), (Kartseva,2004) have developed a methodology and an ontology called e3-value in order to define value models that support the business processes. The e3-value model was developed to support e-commerce business and is essentially focused on the economic value of objects and on activities and actors that create economic value.

Both perspectives of value systems (economic and socio-psychological) are relevant to performance management in collaborative networks, as discussed in (Macedo,2006). In essence the economic perspective provides a transaction mechanism between partners, assuring an equality utility between objects exchanged, and the psychosocial perspective provides a regulation mechanism to ensure social cohesion, to avoid and solve conflicts and to build performance indicators.

\section{METHODOLOGY ADOPTED TO MEASURE THE LEVEL OF VALUE SYSTEM'S ALIGNMENT IN A VBE CONTEXT}

The existence of a VO breeding environment (VBE) is assumed at the basis of the following discussion. A VBE represents an association or pool of organizations and their related supporting institutions that have both the potential and the will to collaborate with each other through the establishment of a "base" long-term cooperation agreement. Whenever a business opportunity is identified by one member (acting as a broker) a subset of these organizations can be selected and thus forming a VO. Various VOs can coexist at the same time in the context of a VBE. A breeding environment, being a long-term networked structure, presents the adequate base environment for the establishment of collaboration agreements, common infrastructures, common ontologies, and mutual trust, which are the necessary facilitating elements when building a new VO (CamarinhaMatos,2003), (Camarinha-Matos,2004). Furthermore, a sustainable VBE should have defined a common value system that "guides" its behavior as a whole. In other words, VBE represents a group of organizational entities that have developed some preparedness for collaboration, in case a specific opportunity arises. Industry clusters or industry districts are examples of such breeding environments.

In this context, a low level of alignment of the value systems of individual members of the VBE is likely to constitute an obstacle for the sustainability of the collaboration, namely in the case of incompatible values. It is therefore important to develop tools that allow estimating the level of alignment of value systems, from different perspectives, for all members. Considering the nature of the concepts here involved, a combination of various "soft modeling" approaches is suggested, as suggested, as shown in Fig. 1. 


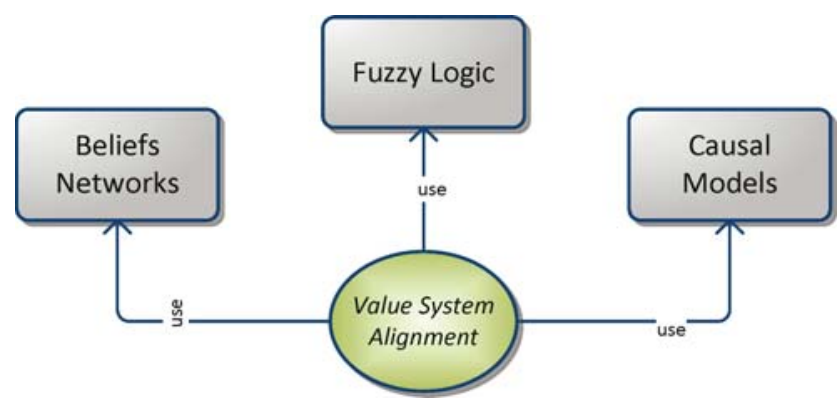

Figure 1. Suggested soft modeling techniques

The Causal Models approach can be applied to analyze the relations of influence between values (Greenland,2002). Based on this approach the level of alignment can be measured in terms of the structural similarity or inter-relationships between Value Systems.

Fuzzy Logic is considered to allow different degrees of inclusion/relevance of each element in a given set (Berthold,2003), it means to allow one element to belong to a given set with a bigger or smaller intensity, also known as degree of membership or degree of truth. Fuzzy reasoning also provides methods to "assemble" partial information, so in this case the partial information about compatible values and incompatible values is used in order to reason about the alignment between Value Systems.

Belief Networks capture relations (which may be uncertain, ambiguous, or imprecise) between a set of variables. Based on the set of states that each variable can have and its belief level (prior probability) it is possible to infer how the prior probability is updated by new data items (Jensen, 1996).

In this case, Beliefs Networks will be used to infer if the values of one partner, based on past behaviors, are aligned with the expected values of another partner.

\section{MODELING EXAMPLES AND RESULTS}

Since the concept of "alignment" is still ill-defined and shows a multifaceted nature, there are several aspects that can be considered when we try to measure the level of alignment between two value systems. In this paper we discuss three perspectives of alignment:

- Perspective 1 - Type of relations among values.

- Perspective 2 - Level of compatibility and incompatibility between value systems.

- Perspective 3 - Members' past behaviors.

\section{Perspective 1}

In collaborative processes, the type of relationships between values can be seen as the seed and ingredient of a successfully co-working. Considering the premises that a member's behavior depends on:

- the way its main values are related, and/or

- potential partners having strategic values that make it consider as advantageous collaborating with them (i.e. values that provide positive impact on its own values).

Under this assumption the level of alignment could be measured in terms of the structural similarity or impact inter-relationships between value systems. For this purpose causal models can be used to model relationships among values. 
In order to illustrate this idea, let us consider the following cases:

Case 1. Let us suppose there are two members in a VO, each one possessing a specific set of main values. Applying causal models the inter-relationship among values could be established and then for each causal model drawn a matrix of influence among values can be defined as shown in Figure 2.
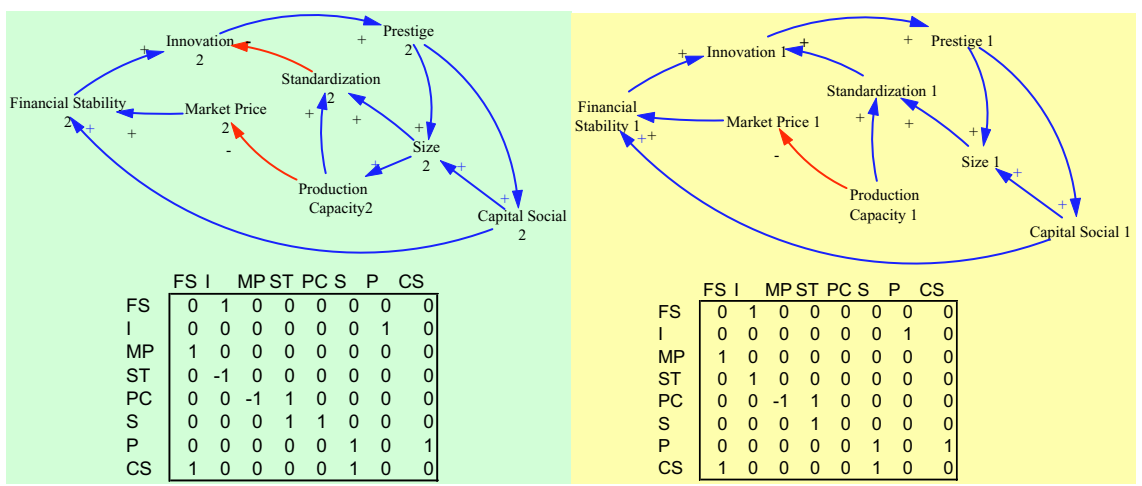

Figure 2. Influence relationships among values

Applying the concept of Euclidean distance we can consider the level of alignment as a metric of structural equivalence. According to this perspective the level of alignment is total if the two value systems are structurally equivalent. In this case the entries in the respective rows and columns of each matrix will be identical, and thus the Euclidean distance between them will be equal to 0 . On the other hand, if they are not structurally equivalent, the level of alignment decreases with the increase of the Euclidian distance.

However, this "understanding" of alignment fails when two actors, although having several common values, they believe only one can maximize its common values through a collaborative process. As such no collaborative process can emerge based on these values. In these cases, other criteria must be used, such as based on impacts between values as discussed in case 2 .

Case 2. Let us suppose two members identified their sets of values and, by applying causal models they defined the impact inter-relationships between value systems. Based on these relations each one builds a matrix of impacts. Figure 3 illustrates the interrelationships between values of two members and the matrix of impacts from the perspective of the industry member. In this example, the level of knowledge of the university can have a (positive) impact on the technological capacity of the industry when they collaborate. 


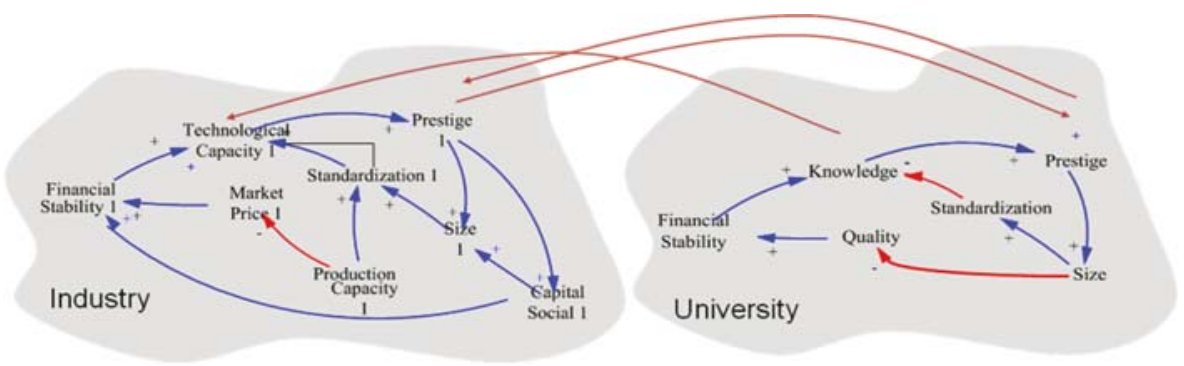

\begin{tabular}{|c|c|c|c|c|c|c|c|c|c|}
\hline \multirow{8}{*}{ 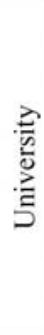 } & & \multicolumn{8}{|c|}{ Industry } \\
\hline & & FS & TC & MP & $\mathrm{S}$ & PC & P & $\mathrm{S}$ & $\mathrm{CS}$ \\
\hline & FS & 0 & 0 & 0 & 0 & 0 & 0 & 0 & 0 \\
\hline & $\mathrm{K}$ & 0 & 122 & 0 & 0 & 0 & 0 & 0 & 0 \\
\hline & Q & 0 & 0 & 0 & 0 & 0 & 0 & 0 & 0 \\
\hline & $\mathrm{S}$ & 0 & 0 & 0 & 0 & 0 & 0 & 0 & 0 \\
\hline & $\mathrm{P}$ & 0 & 0 & 0 & 0 & 0 & I56 & 0 & 0 \\
\hline & $\mathrm{S}$ & 0 & 0 & 0 & 0 & 0 & 0 & 0 & 0 \\
\hline
\end{tabular}

Figure 3. Impact inter-relationships between values systems

Based on this perspective, there is high level of alignment and a potential motivation to collaborate if the sum of impacts is positive and large. On the other hand, if the sum of impacts is null or negative the level of alignment is weak.

\section{Perspective 2}

Let us suppose there are two members in a VO, each one of them possessing its own set of main values as illustrated in Fig. 4.

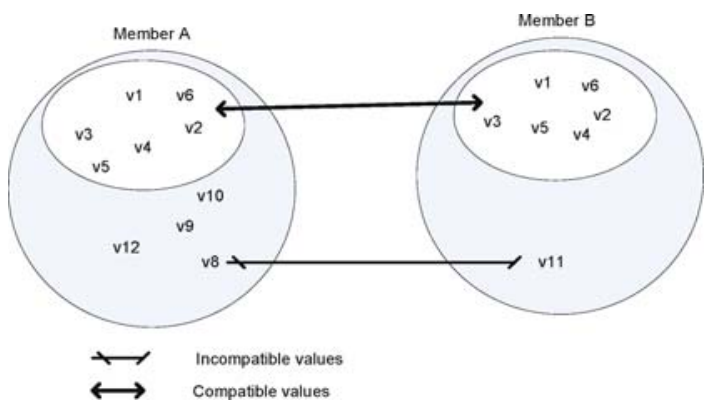

Figure 4. Compatible and incompatible values

As the perceptions of compatible and incompatible values are qualitative and deal with imprecise quantities, one approach is to apply fuzzy logic concepts. Fuzzy Logic can provide methods to "assemble" or reason about the partial information about compatible and incompatible values in order to reason about the alignment level of the value system of two members. 
In order to illustrate how fuzzy logic can be applied in this scenario, let us consider the following table:

Table 1. An example

\begin{tabular}{|l|c|c|c|c|}
\hline $\begin{array}{c}\text { Member A in } \\
\text { relation to B }\end{array}$ & $\begin{array}{c}\text { Compatible } \\
\text { Values }\end{array}$ & $\begin{array}{c}\text { Incompatible } \\
\text { Values }\end{array}$ & $\begin{array}{c}\text { Indifferent } \\
\text { Values }\end{array}$ & $\begin{array}{c}\text { Total Number } \\
\text { of Values }\end{array}$ \\
\hline Total & 6 & 1 & 3 & 10 \\
\hline Percentage & $60 \%$ & $10 \%$ & $30 \%$ & $100 \%$ \\
\hline
\end{tabular}

The concepts of Incompatible Values, Compatible Values and Alignment Level are modeled in a fuzzy way as shown in the Fig. 5. The two variables being analyzed can belong to various fuzzy sets with distinct membership degrees. For instance, $60 \%$ of the number of Compatible Values would be classified as "a lot" with membership of 0.6, and as "most" with membership of 0.4 .
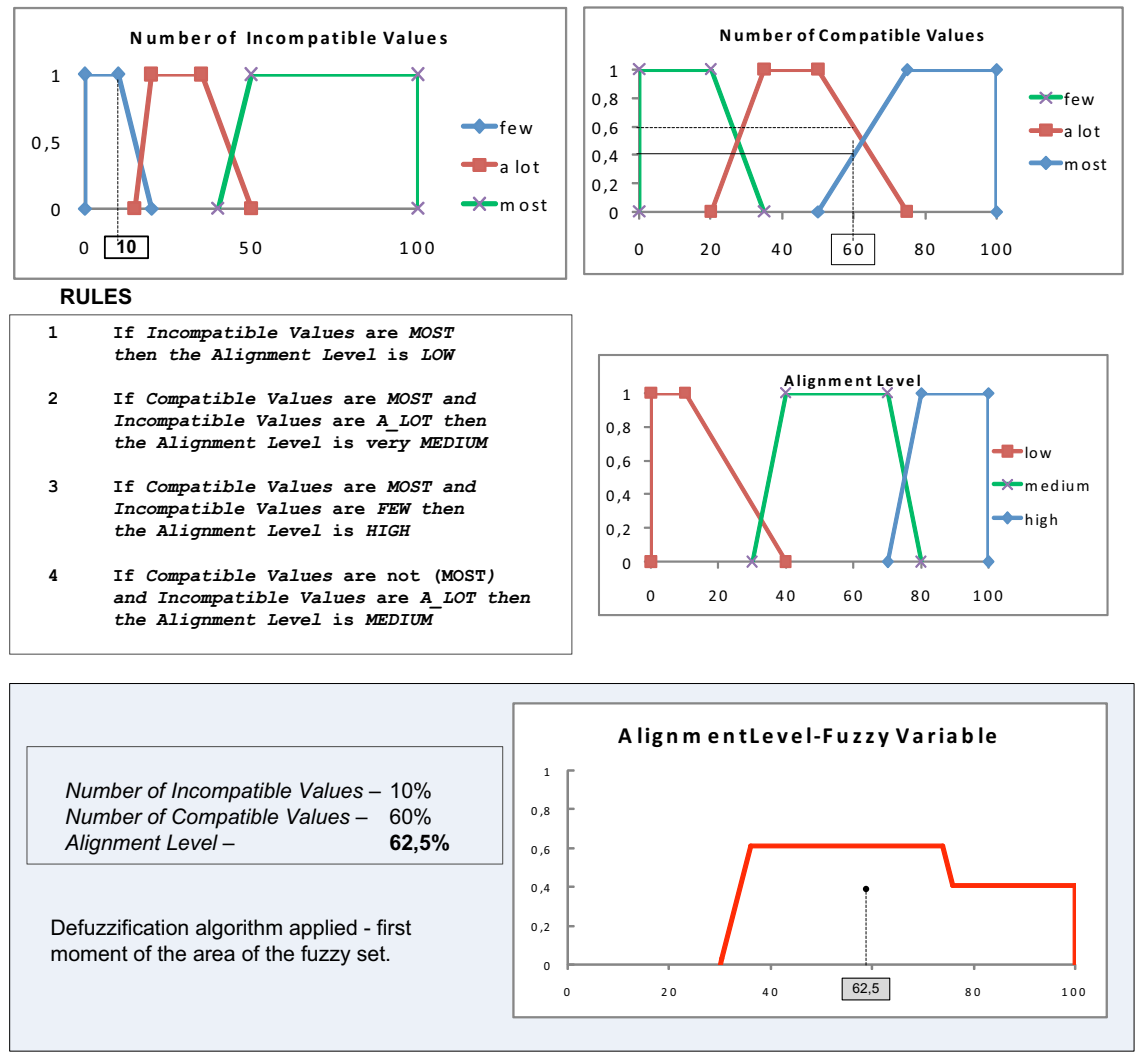

Figure 5. Fuzzy inference process

The Fig. 5 shows a simplified illustration of a fuzzy inference process which is composed of:

- Fuzzification process - Operation through which numeric values are translated into fuzzy sets. In this example there are two cases of fuzzification: Compatible-Values and Incompatible-Values, both modeled by a membership function as it is shown in the figure. 
- Fuzzy Inference- Application of a set of inference rules. In this illustrative scenario, the inference rule associated is of the form: IF Compatible Values are ....AND Incompatible Values are ... THEN the Alignment level is ...... Four inference rules were defined for illustrative purposes.

- Defuzzification process - Operation through which the output linguistic value, induced by the fuzzy inference is translated into a numeric value (Moment defuzzification algorithm was applied) (Kosko,1997). In this example, this operation is modeled by the membership function Alignment-Level as shown in the Figure 5.

In this example the alignment level calculated by the fuzzy inference process is $62 \%$ and resulted from the execution of the rules 3 and 4 .

\section{Perspective 3}

Let us assume that it is possible to establish a connection between past and future behaviors, the behavior of an actor is related with its value system and no explicit representation of the value system is available.

Based on this perspective, the level of alignment can be "measured" through an inference process applying belief networks. In order to illustrate how belief networks can be applied in a VBE context, let us consider the following assumptions:

- There are records of members' past behavior at the VBE management level.

- The partners behavior is explained in terms of a set of values previously defined (principles or qualities considered worthwhile or desirable). There are a finite number of possible values that can describe a partner's behavior. For instance:

Partner behavior $=<$ Quality, Prestige, Lead time $>$

- There are a finite number of possible states associated to each value that can be chosen to measure the value.

- Each value can have a set of states and its belief level (prior probability) is expressed in terms of percentage based on past cooperative behaviors.

Let us assume an enterprise B needs to establish a network where Quality and Lead Time are two strategic values. In order to avoid undesirable partners, it is important to identify members that have a strong alignment with it (according to B's evaluation) in relation to these two values.

Based on past experiences with others partners in collaborative processes, when an enterprise B identifies another one that considered quality and lead time as strategic values, the probability that such partner in the future adopts these values as strategic, according to the beliefs of $\mathrm{B}$, is $90 \%$ (prior probabilities) as shown in table 2 .

Based on past records an enterprise A exhibited the following behavior in terms of quality:

- $60 \%$ of the times is considered a strategic value

- $40 \%$ of the times is considered an irrelevant value; and, in terms of lead time:

- $70 \%$ of the times is considered a strategic value

- $30 \%$ of the times are considered an irrelevant value. 
Table 2. Beliefs of B based on past experiences

\begin{tabular}{|c|l|c|c|c|}
\hline \multicolumn{2}{|c|}{ Values } & \multicolumn{3}{|c|}{ Level of Alignment } \\
\hline Quality & Lead Time & Strong & Medium & Low \\
\hline \multirow{2}{*}{ Strategic } & Strategic & 0,9 & 0,1 & 0 \\
\cline { 3 - 5 } & Irrelevant & 0,6 & 0,3 & 0,1 \\
\hline \multirow{2}{*}{ Irrelevant } & Strategic & 0,2 & 0,5 & 0,3 \\
\cline { 2 - 5 } & Irrelevant & 0,2 & 0,3 & 0,5 \\
\hline
\end{tabular}

Applying Belief Networks for these two values, enterprise B can infer the Level of Alignment in relation to partner A, as shown in Figure 6.

Base on the available data, the enterprise B infers there is a probability of $56,6 \%$ that A will adopt a behavior that considers both values strategic.

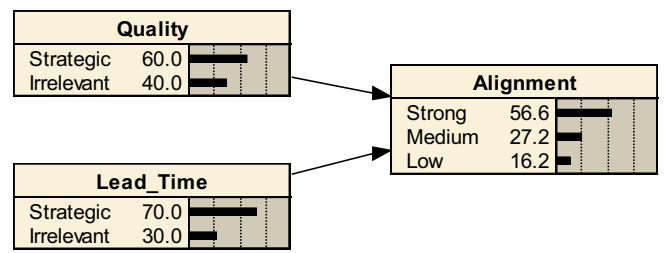

Figure 6. Alignment level inference

\section{CONCLUSIONS}

The measurement of the alignment level of value systems can be a useful instrument to the VBE manager, to a VO broker, and to a VBE member. This indicator can be determined for a particular collaboration process (a particular VO occurrence) or over a period of time (average values) and can be used in decision-making processes, such as the planning of a new VO. However the concept of "alignment" can have different interpretations. Therefore, various perspectives have been discussed, using different soft modeling approaches.

Some benefits of the proposed modeling approaches can be summarized as:

- The causal models are easy to understand and useful for describing in a simplified way the value system (its structure and cause-effect relations), and using simple calculus it is possible to reason about the level of alignment.

- The belief networks and fuzzy logic models have the advantage of dealing well with imprecise and partial information.

- The fuzzy logic models allow measuring the level of alignment through a linguistic form.

- The belief networks can be useful to predict the level of alignment based on past behaviors.

However, the proposed models also carry some limitations such as:

- For large problems, the collection of information to build a model can be very difficult.

- The models are not easy to maintain and modify if proper tools are not available.

- In case of causal models, if there are many interdependencies between values the calculation becomes very time consuming.

- In real application, belief networks demand a record of past behavior that might not be available. 
Furthermore, to reason about the value system alignment other approaches might be useful, such as two-sorted logic, par consistent logics, and dynamic systems. Consequently the development of methodologies to measure the value systems alignment in collaborative networks still requires further work. Some preliminary steps in this direction were presented and initial results illustrate the applicability of the suggested approach.

\subsection{Acknowledgments}

This work as supported in part by the ECOLEAD integrated project funded by the European Commission.

\section{REFERENCES}

1. Abreu A, Camarinha-Matos LM. "On the Role of Value Systems and Reciprocity in Collaborative Environments". In Network-Centric Collaboration and Supporting Frameworks, Spring, ed. IFIP, Volume 224: Boston Springer, 2006.

2. Berthold MR. Intelligent Data Analysis: An Introduction. Springer, 2003.

3. Camarinha-Matos LM, Afsarmanesh (Ed.s) H. Collaborative Networked Organizations - A research agenda for emerging business models: Kluwer Academic Publishers, 2004.

4. Camarinha-Matos LM, Afsarmanesh H. "Elements of a VE base infrastructure". J. Computers in Industry 2003;51:139-163.

5. Filipe J. "The organizational semeiotics normative paradigm". In Collaborative Networked Organizations. London: Springer, 2003: 261-272.

6. Goguen J. "Semiotics, compassion and value-centered design". In Keynote lecture, in Proceedings of the Organizational Semiotics Workshop, University of Reading; UK, 2003.

7. Gordijn J, J.M. Akkermans, Vliet JCv. "Value based requirements creation for electronic commerce applications". In 33rd Hawaii International Conference on System Sciences; Hawai, 2000.

8. Greenland S, Brumback B. "An overview of relations among causal modeling methods". International journal of epidemiology, 2002.

9. Hartman R. The Measurement of Value. In; 1973, 1973.

10. Kartseva V, Gordijn J, Akkermans H. "A Design Perspective on Networked Business Models: A Study of Distributed Generation in the Power Industry Sector". In 12th European Conference on Information Systems; 2004.

11. Kosko B. Fuzzy Engineering. New Jersey: Prentice Hall, 1997.

12. Macedo P, Sapateiro C, Filipe J. "Distinct Approaches to Value Systems in Collaborative Networks Environments". In Network-Centric Collaboration and Supporting Frameworks: Springer Boston, 2006: 111-120.

13. Mefford D, Meffortd V. Values Usage Exercise (VUE) A Tool For Raising Values Awareness Concerning The Professional - Personal Values Interface. In: Conference on Professional Ethics. WASHINGTON, 1997.

14. Merton RK. Social Theory and Social Structure. The Free Press 1957;3.

15. Rokeach M. The nature of human values. New York: Free Press. 1973.

16. Tan Y-H, Thoen W, Gordijn J. "Modeling Controls for Dynamic Value Exchange in Virtual Organizations". In Berlin S, ed. Trust Management, 2004: 236-250.

17. Woods J. Paradox and Para consistency: Conflict Resolution in the Abstract Sciences. Cambridge: Cambridge University Press, 2003. 